\title{
The video-recorder in the accident and emergency department
}

\author{
L. MURRAY \& M. MCCABE
}

Accident \& Emergency Department, Cardiff Royal Infirmary, Longcross Street, Cardiff

\section{INTRODUCTION}

Video recording is widely used for teaching purposes. In Psychiatry and in General Practice it is used to assess interpersonal and non-verbal communications which are often difficult to monitor by other means. Video-recording of major trauma and emergency care is widely practiced in the United States (Peltier et al., 1969; Hoyt et al., 1988). Optimum care of these patients requires rapid assessment and treatment and investigation with a correct order of priorities. Other than by stationing a skilled observer in the reception area with notebook and stop-watch $\vec{\theta}$ video recording offers the only way to assess whether these objectives are obtaines

\section{MATERIALS AND METHODS}

We fitted a colour Panasonic video-camera which uses standard VHS cassettes in our main resuscitation room. It also recorded sound through an integral or remote microphone. Pictures can be replayed through a standard video recorder. The cost of such equipment has fallen in the 2 years since we started this experiment, and it could now be established for around $£ 500$.

With the camera set for wide-angle filming it can capture almost the entire room. It has been mounted on a camera mount and boxed in for security purposes. It films through a one way mirror. The camera is positioned behind the patient's head to maintain anonymity.

We undertook recordings either randomly by leaving the camera running, or by $\frac{7}{0}$ a Staff Nurse or Doctor starting recording shortly prior to an anticipated arrival. The camera would run for a maximum of $4 \mathrm{~h}$.

Correspondence: Leo Murray, Accident and Emergency Department, Cardiff Royal Infirmary, Longcross Street, Cardiff CF2 1SZ, Wales. 


\section{RESULTS}

\section{Reviews of films}

Films were reviewed by our Consultants and Senior Registrars, and subsequently reviewed with our SHOs during audit and teaching sessions.

We studied whether the correct order of priorities, was observed, and how long it took to establish control of airway and breathing, place IV lines, send blood samples, and obtain initial X-rays. The camera is set to automatically date and time stamp and so it is easy to establish how long it takes for personnel to attend, and to time clinical procedures.

\section{What did we find?}

We noted first that priorities were not being observed, and that initial control took much longer than we hoped. Coordinating radiography with resuscitation was a problem. While anaesthetic response was rapid, orthopaedic and surgical response was often greatly delayed.

However, after reviewing films with the SHOs over each 6-month period, a noticeable improvement in prioritization, and time to undertake basic manouevres, was obtained. The importance of applying arm traction to obtain satisfactory cervical spine $X$-rays in the first instance, rather than waiting to see an initial unsatisfactory plate, was a simple lesson.

We observed that a great deal of movement within the resuscitation area to obtain equipment could be avoided by simply repositioning stocks. The dearth of power points and the consequent crowding of monitors and leads caused an obstruction, and additional points were installed. More difficult to address was the realization that our layout of oxygen and suction points forced us to treat patients in an alignment which obstructed the flow of personnel. In addition the essential need for a spacious resuscitation area, where many people are inevitably working simultaneously, could not have been more clearly demonstrated. The value of a clearly visible stop-clock was rapidly apparent, and one was installed.

\section{DISCUSSION}

\section{Acceptance of video-recording}

Video-recording, although increasingly widespread, is often disliked and resented as a 'spy on the wall'. The question of consent by the patient, obviously not feasible in a major resuscitation, remains unresolved. Reiuctance on the part of both medical and nursing staff to participate in our experiment was however allayed after much discussion. Guidelines limiting the viewing of the material to initial review by senior medical staff, and then structured and guided viewing with SHOs, were agreed. The erasure of recorded material within 1 week was 
accepted. This inevitably restricts sequential comparison of material, and limited $\stackrel{\oplus}{\stackrel{\oplus}{\oplus}}$ formal study, but had to be accepted to gain staff cooperation.

\section{Benefits}

The relative scarcity of major trauma seen in our hospital means that any one individual SHO will see only one or two such incidents during their 6-month attachment. The video-recordings enabled a sharing of such experience and this $\mathbb{\otimes}$ produced a definite improvement in performance. The alterations to the layout of our resuscitation room as a result have improved the speed and efficiency of our resuscitations.

\section{Future plans}

Because the camera is not primarily designed for surveillance, switching it on $\stackrel{\dot{\omega}}{\dot{\omega}}$ requires several manipulations high up in its mounting box. We now intend to $\vec{\sim}$ install a dedicated surveillance camera, remote from the recording equipment, $\circ$

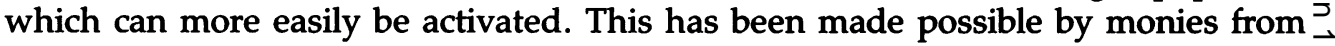
the Welsh Office, from funds allocated for audit projects. This system will consist 0 of an Ikegami CV770 Camera, with power supply, $8 \mathrm{~mm}$ lens and bracket, a Baxall $\frac{\mathbb{C}}{\mathbb{D}}$ Time and Date generator, a JVC HRD660 long play VHS recorder and sensitive plate microphone - cost about $£ 1500$.

\section{REFERENCES}

Peltier L. F., Geertsma R. H. \& Youmans R. L. (1969) Television Videotape Recording: An adjunct in teaching Emergency Medical Care. Surgery 66, 233-236.

Hoyt D. B., Shackford S. R., Fridlnd D. H. et al. (1988) Videorecording Trauma Resuscitations: an effective teaching technique. The Journal of Trauma 28, 435-440. 